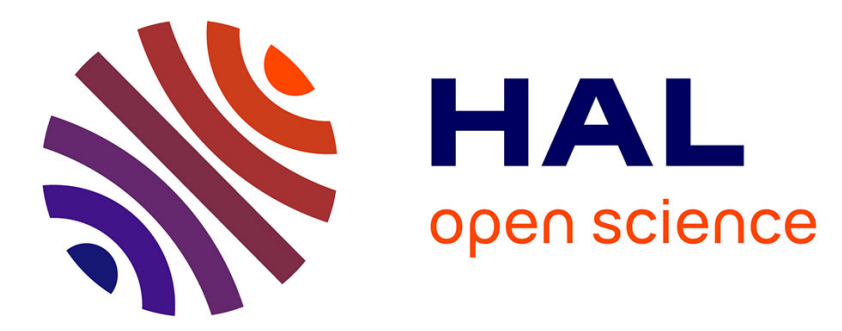

\title{
Mapping the nonlinear dynamics of a laser diode via its terminal voltage
}

\author{
Aakash A Sahai, Byungchil Kim, Daeyoung Choi, A. Locquet, D. S Citrin
}

\section{To cite this version:}

Aakash A Sahai, Byungchil Kim, Daeyoung Choi, A. Locquet, D. S Citrin. Mapping the nonlinear dynamics of a laser diode via its terminal voltage. Optics Letters, 2014, 39 (19), pp.5630. 10.1364/OL.39.005630 . hal-03079713

\section{HAL Id: hal-03079713 https://hal.science/hal-03079713}

Submitted on 17 Dec 2020

HAL is a multi-disciplinary open access archive for the deposit and dissemination of scientific research documents, whether they are published or not. The documents may come from teaching and research institutions in France or abroad, or from public or private research centers.
L'archive ouverte pluridisciplinaire HAL, est destinée au dépôt et à la diffusion de documents scientifiques de niveau recherche, publiés ou non, émanant des établissements d'enseignement et de recherche français ou étrangers, des laboratoires publics ou privés. 
To be published in Optics Letters:

Title: $\quad$ Mapping the nonlinear dynamics of a laser diode via its terminal voltage

Authors: Aakash Sahai, David Citrin, Byungchil Kim, Alexandre Locquet, and Daeyoung Choi

Accepted: 8 August 2014

Posted: 22 August 2014

Doc. ID: 217102 


\title{
Mapping the nonlinear dynamics of a laser diode via its terminal voltage
}

\author{
Aakash A. Sahai, Byungchil Kim, Daeyoung Choi, A. Locquet, D. S. Citrin ${ }^{1}$ \\ ${ }^{1}$ School of Electrical and Computer Engineering, Georgia Institute of Technology, \\ Atlanta, Georgia 30332-0250 and UMI 2958 Georgia Tech-CNRS, \\ Georgia Tech Lorraine, 2 Rue Marconi, F-57070, Metz, France
}

compiled: August 20, 2014

\begin{abstract}
We show that the bifurcations between dynamical states originating in the nonlinear dynamics of an external-cavity semiconductor laser at constant current can be detected by its terminal voltage $V$. We experimentally vary the intensity fed back into the gain medium by the external cavity and show that the dc component $V_{\mathrm{dc}}$ of $V$ tracks the optical intensity based bifurcation diagram. It is shown using computational results based upon the Lang-Kobayashi model that whereas optical intensity accesses the dynamical-state variable $|E|, V$ is related to population-inversion carrier density $N$. The change in feedback strength affects $N$ and thereby the quasi-Fermi energy level difference at the $p-i-n$ junction band-gap of the gain medium. The change in the quasi-Fermi energy-level thereby changes the terminal voltage $V$. Thus $V$ is shown to provide information on the change in the dynamical-state variable $N$, which complements the more conventionally probed optical intensity.
\end{abstract}

Laser diodes (LD) driven at constant current are often exposed to optical feedback from external optical components. This time-delayed feedback incites nonlinear dynamics observed as instability of emitted light with extreme sensitivity to operating parameters. Conventionally the dynamics is studied for various applications [1] by sampling the optical output. In this letter we present an effect where the voltage $V$ across an external-cavity semiconductor laser (ECSL) complements the study of chaotic dynamics accessed by the time-resolved optical output. We vary the intensity fed back into the gain medium by the external cavity and show that $V$ tracks the optically measured bifurcations between different dynamical states. Applications of terminal voltage $V$ across a laser diode [2, 3] have been explored in the negligible feedback linear-regime of the ECSL phase-space. However, the non-linear dependence of the terminal voltage on the feedback level due to abrupt transitions of the dynamical state across different regions of the phase-space has been ignored. It is shown here that in the non-linear regime, for a negligible instantaneous change in optical feedback, $N$ changes significantly at the bifurcations corresponding to jumps of phase-space trajectory across several external cavity spatial modes (ECM). The quasi-Fermi level bandgap potential difference across the $p-i$ - $n$ active-medium changes proportional to change in $N$.

An understanding of non-linear instability of the dynamical state of the ECSL is critical because the effects of feedback are not understandable in terms of perturbation about a given ECM. It is well known both experimentally [4-8] and theoretically [9] that ECSLs exhibit a rich variety of non-linearity, including fully-developed chaos, when varying operating parameters such as the feedback strength. The feedback-to-voltage physics in Ref. [2, 3, 10, 11] does not take account of the rich dynamics. We show that the dynamical regimes-as well as the bifurcations between them-are reproduced in the dc component $V_{\mathrm{dc}}$ of $V$ while tracing the more typically detected optical output. The changes in feedback intensity modify the stimulation-rate controlling the population inversion that affects the quasi-Fermi energylevels and thereby $V$. In other words, we directly access a key dynamical-state variable, the inversion density $N$, which complements the knowledge of the system from conventionally probed state variable $|E|$, accessed through optical intensity fluctuations $\delta \mathcal{I}$. Importantly, $V$ is an electrical signal and thus a photodetector is not required in contrast to accessing the optical intensity $\mathcal{I}$. The experimental results based on the terminal voltage are shown to be in excellent agreement with the optically detected signal and explained by the simple physical model presented here. Our results, therefore, encompass observations discussed in Ref. [12, 14] where $V$ was used to characterize certain dynamical characteristics. But, in our work we show the significance of terminal voltage as a dynamical state detector over the entire phase-space.

The experimental setup is shown in Fig. 1. The intrinsically single-longitudinal-mode edge-emitting InGaAsP DFB laser used as the gain medium oscillates at $1550 \mathrm{~nm}$ and maximum $\mathrm{cw}$ power of $15 \mathrm{~mW}$. The freerunning threshold current $I_{\text {th }}$ is $9.27 \mathrm{~mA}$. $T$ and $I$ controllers maintain constant $T(<0.002 \mathrm{~K} /$ day $)$ and $I(<100$ $\mu \mathrm{A} /$ day). In order to detect changes in $V_{\mathrm{dc}}$, a multimeter and an oscilloscope are connected to the LD voltage terminals. The external cavity length $L$ is set to 15 and $65 \mathrm{~cm}$, with external-cavity round-trip time $\tau=1$ and 


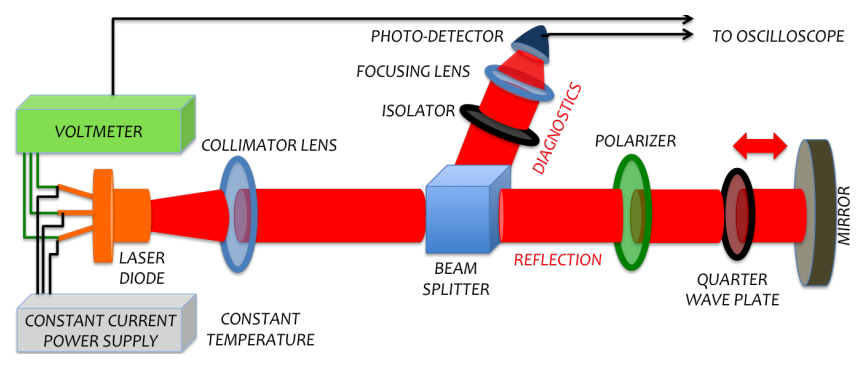

Fig. 1: Experimental setup. A laser diode (LD) is powered with a constantcurrent power supply (at constant temperature) and its dc terminal voltage $V_{\mathrm{dc}}$ is monitored on a voltmeter and oscilloscope. The laser output is linearly polarized and split with one path being for external reflection to form an external cavity and the other arm being for diagnostics. The diagnostics are isolated from the dynamical system using a unidirectional optical isolator.

$4.3 \mathrm{~ns}$, respectively. In this Letter, $\sim 16 \%$ of the optical power is fed back onto the collimating lens at maximum experimental feedback ratio $\eta=0.8$. A motorized piezo-actuation stage adjusts the angle of the quarterwave plate relative to the polarizer, controlling $\eta$. The diagnostics optical signal is sent to a photodetector and intensity $\mathcal{I}(t)$ fluctuations are detected.

We present the experimental results in Fig. 2 where the optical $\mathrm{BD}$, corresponding dc terminal voltage $V_{\mathrm{dc}}$ and LD-internal photodiode signal $P_{P D}$ equivalent to the average intensity $\langle\mathcal{I}\rangle$ from the leakage sensed at the back-facet of the optical cavity, are shown as functions of the feedback strength $\eta$ for a given $L$ and injection current $I$. The BD in Fig. 2(a), (d) is obtained by detecting the time-series of $\mathcal{I}(t)$ envelope fluctuations and plotting the histogram of local extremal values for a given $\eta$; probability density is highest in white and progressively darker (blue in the color figure) in low probability density regions.

Bifurcation cascades between apparently stable and unstable regions are observed in Fig. 2(a), (d) as changes in $\delta \mathcal{I}$ with varying $\eta[7]$. The stable thinner regions of $\delta I$, do not necessarily correspond to stable $C W$ behavior but also contain regimes in which phase-space trajectories have small excursions within an island of stability resulting in low-amplitude instabilities that have developed around a single ECM attractor [15]. The wider regions in $\delta \mathcal{I}$, referred to as unstable regions, typically correspond to regimes in which phase-space trajectories wander around several and occasionally non-adjacent ECMs and are thus above the system noise. Unsurprisingly while $\langle\mathcal{I}\rangle$ in Fig. 2(c), (f) also shows the bifurcations in $\delta \mathcal{I}$, it has an overall rising trend for increasing $\eta$ due to a rising stimulated emissions rate.

The differences between Fig. 2(a)-(c) and 2(d)-(f) can largely be attributed to the frequency separation between ECMs. When $L$ is long $(65 \mathrm{~cm})$, the optical spectral separation between ECMs is reduced (for $L=15$ $\mathrm{cm}$, the external-cavity free spectral range is $1 \mathrm{GHz}$, for $L=65 \mathrm{~cm}, 233 \mathrm{MHz}$ ). Therefore, each participating mode being closer in phase space, large-amplitude itinerancy between several modes is easily observed. Indeed, numerical simulations of the trajectories on the ellipse in phase space projected on the $N-\varphi$ phase plane show that the proximity of the ECMs for longer cavities impedes the development of independent attractors and thus prevents bifurcation cascade. Conversely, for shorter $L$ cavities, the increased separation between adjacent ECMs means that larger $\eta$ is needed before the attractors merge, thus explaining the longer stable regions.

In Fig. 2(b), (e) voltage change $\delta V_{\mathrm{dc}}$ is plotted as $\eta$ is varied; these correspond to the optical-BDs in Fig.2(a), (d), respectively. We note an overall (though clearly not monotonic) decrease in $\delta V_{\mathrm{dc}}$ with increasing $\eta$; various bifurcations that occur in $\delta \mathcal{I}(t)$ in Fig. 2(a), (d) at specific values of $\eta$ are clearly reflected at the same $\eta$ in the terminal voltage $\delta V_{\mathrm{dc}}$ in Fig. 2(b), (e). This juxtaposition of $\delta I$ with $\delta V_{\mathrm{dc}}$ and a clear trend in $\delta V_{\mathrm{dc}}$ opposite to $\langle\mathcal{I}\rangle$ illustrates the ability to reliably tap into additional dimensions of phase space but without a photo-detector.
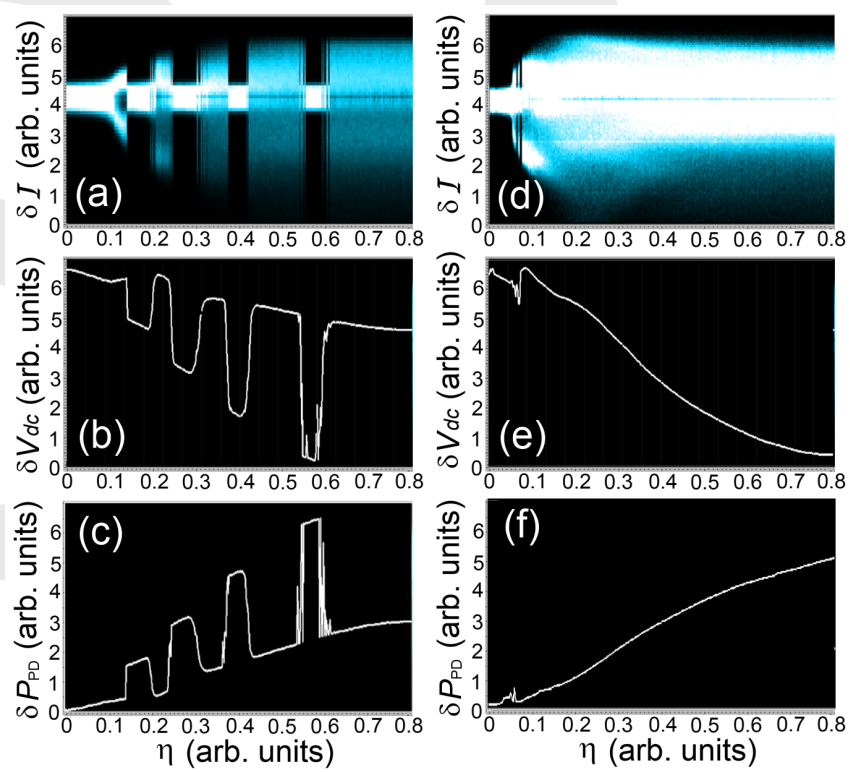

Fig. 2: Experimental optical-BD, corresponding $\delta V_{\mathrm{dc}}$ and $\langle\mathcal{I}\rangle$ from $P_{P D}$ the back-facet optical leakage sensed by the internal photo-diode (a), (b), (c) for $I=$ $11.42 \mathrm{~mA}$ and $L=15 \mathrm{~cm}$, with a frequency spacing between ECMs of $\sim 1 \mathrm{GHz}$. (d), (e), (f) for $I=11.42 \mathrm{~mA}$ and $L=65 \mathrm{~cm}$, with a frequency spacing between ECMs of $\sim 233 \mathrm{MHz}$.

We next turn to a theoretical description of the nonlinear dynamics of the ECSL. The Lang-Kobayashi (LK) model is a single-longitudinal-mode description of ECSL in terms of rate equations [9]. This approach integrates out spatial degrees of freedom; nonetheless, while obtaining perfect agreement between theory and experiment is not expected, the LK equations reliably predict dynamical trends as a function of various pa- 
rameters [9]. The LK equations are [5]

$$
\begin{aligned}
\frac{d E}{d t} & =\frac{1}{2}(1+i \alpha)\left(\mathcal{G}-\tau_{p}^{-1}\right) E(t)+\frac{\kappa}{\tau_{i n}} E(t-\tau) e^{-i \omega_{0} \tau}+F_{E}(1) \\
\frac{d N}{d t} & =p J_{t h}-\frac{N(t)}{\tau_{s}}-\mathcal{G}|E|^{2} .
\end{aligned}
$$

where $E(t)$ is the slowly-varying envelope of the complex optical field $\left(|E|^{2}\right.$ is the photon number in unit active-region volume), $N(t)$ is the carrier density in the active region, $\mathcal{G}=g\left[N(t)-N_{0}\right]$ is the modal gain (in $\mathrm{s}^{-1}$ ) where $g$ is the differential modal gain coefficient (in $\mathrm{m}^{3} \mathrm{~s}^{-1}$ ), and $N_{0}$ the carrier density at transparency. $\tau_{p}$ is the photon lifetime, $\tau_{s}$ is the carrier lifetime, $\tau_{\text {in }}$ is the round-trip time in the gain medium, $\tau=2 \mathrm{~L} / \mathrm{C}$ is the optical round-trip time in the external cavity (the time delay of the feedback) with $c$ the speed of light, $\alpha$ is the linewidth-enhancement factor, $p$ is the current pumping factor, and $J_{t h}$ is the threshold and, $J=p J_{t h}$ is the injection current areal density. The theoretical feedback strength $\kappa$ is proportional to experimental feedback ratio $\eta$. The spontaneous-emission noise is modeled by an additive term $F_{E}=\sqrt{2 \beta N} \xi$, where $\beta$ is a spontaneous-emission noise factor, and $\xi$ is a complex Gaussian white noise of zero mean and autocovariance function $C_{x}(t)=2 \delta(t)$. We numerically integrate Eqs. (1) and (2) with parameters $g=8.1 \times 10^{-13} \mathrm{~m}^{3} / \mathrm{s}, N_{0}=1.1 \times 10^{24} \mathrm{~m}^{-3}, \tau_{p}=1$ $\mathrm{ps}, \tau_{\text {in }}=8 \mathrm{ps}, \tau_{\mathrm{s}}=1 \mathrm{~ns}, \alpha=3$, and $\omega_{0} \tau=2 \pi v$ for some integer $v[15]$.

Fig. 3(a), (c) show the computational BD (subtracting off the time-averaged $\mathcal{I} \propto|E|^{2}$ ) and Fig. 3(b), (d) the time-averaged carrier density $\delta N_{\mathrm{dc}}$ as a function of $\kappa$ for $L=15$ and $65 \mathrm{~cm}$, respectively. Note that the BDs bear a close resemblance to those of Fig. 2(a), (d) [15]. Importantly, as discussed below, $\delta N_{\mathrm{dc}}$ in Fig 3 (b), (d) is seen equivalent to experimentally measured $\delta V_{\mathrm{dc}}$ in Fig[2(b), (e).

The voltage across the $p-i-n$ structure of a laser diode (LD), $V$ is the difference $\Phi(\mathrm{t})$ between the quasi-Fermi levels $\mathcal{E}_{F}^{j}$ per unit charge, $e$, on the $j=p$ and $n$ extremities of the band-gap. Thus, assuming flat bands in the quasi-neutral regions,

$$
\Phi(t)=\mathcal{E}_{F}^{n}(t)-\mathcal{E}_{F}^{h}(t)=\left[\ln \left(\frac{N_{e}(t)}{N_{e}^{i}(T)}\right)-\ln \left(\frac{N_{h}(t)}{N_{h}^{i}(T)}\right)\right] k_{B} T,
$$

where $N_{e}, N_{h}, N^{i}(T)$ are the electron, hole (in the $n$ and $p$-type regions, respectively), and intrinsic densities [2]. We assume $I$ and $T$ are constant, and the ECSL dynamics fluctuates around one ECM. The change in the carrier density, $\delta N$ due to dynamical-state itinerancy is assumed to be small compared with the gain medium carrier density $N_{\mathrm{ECM}}$ in the quasi-neutral intrinsic region, giving $N(t) \simeq N_{e}(t)=N_{h}(t)=N_{\mathrm{ECM}}$. By measuring the voltage change $\delta V$ in response to the change in $\Phi$ as $N$ changes, we determine the change in $N, \delta N$ due to non-linear dynamics induced by optical-feedback. The expression relating the change in $V_{\mathrm{dc}}$ due to the change
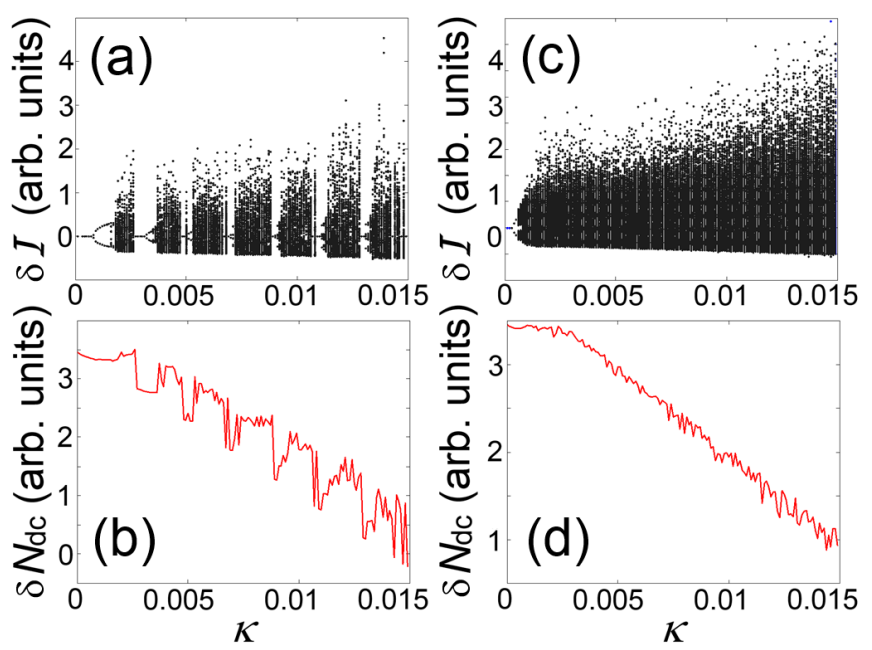

Fig. 3: Theoretical $|E|^{2}-\mathrm{BD}$ and corresponding $\delta N_{\mathrm{dc}}\left(\propto \delta V_{\mathrm{dc}}\right)$ obtained by computational evaluation of Eq. 11- 2. (a), (b) for $p=1.3$ and $L=15 \mathrm{~cm}$. (c), (d) for $p=1.3$ and $L=65 \mathrm{~cm}$.

in the dc value of $N$ is obtained by varying Eq. (3).

$$
\delta V_{\mathrm{dc}}=\frac{\delta \Phi_{\mathrm{dc}}}{e}=\frac{\delta N_{\mathrm{dc}}}{e N_{\mathrm{ECM}}}\left(\frac{\partial \mathcal{E}_{F}^{e}}{\partial \ln N_{e}}-\frac{\partial \mathcal{E}_{F}^{h}}{\partial \ln N_{h}}\right)
$$

where $\delta N_{\mathrm{dc}}=N(t)-N_{i}$ in the gain medium within a given dynamical regime. Since $\partial \mathcal{E}_{F}^{e} / \partial \ln N_{e}>0$ and $\partial \mathcal{E}_{F}^{h} / \partial \ln N_{h}<0$ [16], $\delta V_{\mathrm{dc}}$ is proportional to $\delta N_{\mathrm{dc}}$ and of the same sign. It should be noted that when the ECSL undergoes a bifurcation, $N$ undergoes a discontinuity; however Eq.(4) proportionality holds.

Optical feedback induces a rise in the photon number $\left(\propto|E|^{2}\right)$ in the active region, increasing the stimulation rate, this results in an overall increase in the stimulated recombinations, increasing $\langle\mathcal{I}\rangle$ in Fig.2(c), (f). Increased stimulated recombinations reduce population inversion density $N_{\mathrm{dc}}\left(\dot{N} \propto-|E|^{2}\right)$. Consequently, the overall reduction in $N_{\mathrm{dc}}$ in the active region leads to a reduction in $\Phi_{\mathrm{dc}}[16]$ and thereby in $V_{\mathrm{dc}}$ in Fig. 2(b), (e). While the analysis above describes the time-dependence of the quantities, the voltmeter monitors the average voltage $V_{\mathrm{dc}}$. Nonetheless, the dc voltage analysis provides rich insight into the physics-at a level comparable to the optical BD. Clearly, signatures of the various bifurcations are experimentally observed both in Fig. 2(b), (e) using $V_{\mathrm{dc}}$ and from computational results of $N_{\mathrm{dc}}$ based on LK equations in Fig. 3(b), (d). Based on Eq. (4) in the dc limit, we see that the $\delta N_{\mathrm{dc}}$ curves are proportional to $\delta V_{\mathrm{dc}}$. Namely, the overall downward trend in $\delta V_{\mathrm{dc}}$ and $\delta N_{\mathrm{dc}}$ with increasing feedback ratio $\eta$ is interrupted by abrupt jumps at bifurcations, as seen in the corresponding optical BDs [Fig. 2(a), (d); Fig. 3(a), (c)].

Using Eq. (4) model we compare Fig. 2(a) and (b), (d) and (e) and Fig. 3(b) and (d) respectively, and clearly see a distinct advantage of the terminal-voltage approach in accessing $N$. While $N_{\mathrm{dc}}$ reduces for increasing feed- 
back inferred from Fig. $3(\mathrm{~b})$ and (d), the optical-BD and $\langle\mathcal{I}\rangle$ fail to provide information on the itinerancy of the phase-space trajectories around the ECMs in the $N-\varphi$ plane. For the case of $L=65 \mathrm{~cm}$ (Fig. 2(e), Fig. 3(d)), at fairly low $\kappa$ the ECSL enters a chaotic regime with no clearly discernible state transitions from the optical $\mathrm{BD}$ apart from the initial one at low $\eta$. In contrast, $\delta V_{\mathrm{dc}}$ undergoes a steady decrease in the unstable region revealing the continual drift of the dynamical state of the ECSL towards lower $N$ in the phase-space in the direction of the maximum gain mode.
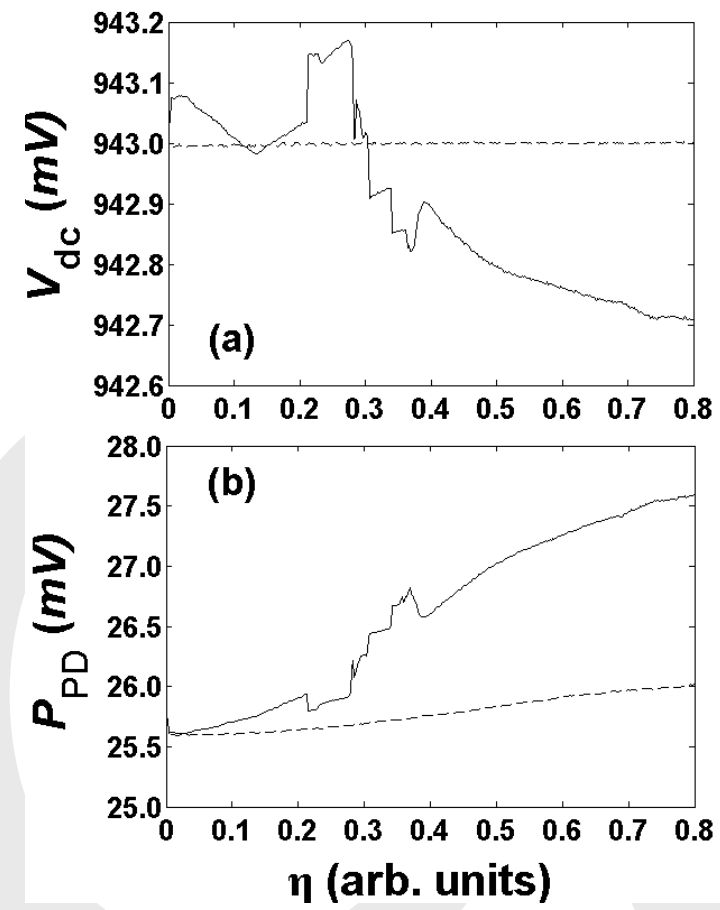

Fig. 4: (a) $V_{\mathrm{dc}}$ and (b) voltage equivalent of $P_{\mathrm{PD}}$ for $I=21.14 \mathrm{~mA}$ and $L=30 \mathrm{~cm}$. The solid curves show the voltage for the optimally aligned external cavity; the dashed curves with the external cavity intentionally misaligned. The dashed curve in (b) shows that $P_{\mathrm{PD}}$ changes with apparent $\eta$ even when the actual feedback into the $\mathrm{LD}$ active medium is negligible.

The non-linear dynamics of ECSL, at the level of the LK model, is characterized by three state variables, the electric-field amplitude $|E(t)|$, the optical phase $\varphi(t)=\arg E(t)$, and the population inversion carrier density $N(t)$. $\mathcal{I}(t)$ and thus $|E(t)|$ is commonly probed. The present work provides information on the dependence of dynamics on $N$, though only the dc value $N_{d c}$. Nonetheless, as shown such information is indeed valuable and provides insight into the dynamics on a level comparable to the optical BD constructed from $\delta \mathcal{I}(t)$. The steady-state solutions of LK model (Eq.(1)-(2)) are the stable ECMs and unstable anti-modes. In the $N-\varphi$ plane, solution fixed-points sit on an ellipse [4]. Thus, measuring $\delta V_{\mathrm{dc}}$ provides information on those ECMs that dominate the phase-space trajectory at different feedback levels. Such measurements can be compared with the optical spectrum [15]. The relevant ECMs across which the phase-space trajectory wanders and jumps can be identified from $V_{\mathrm{dc}}$. Future work will focus on resolving the dynamics of $N(t)$. The remaining state variable, $\varphi(t)$ has attracted less attention, and indeed requires an interferometric approach [7].

We also show that $V_{\mathrm{dc}}$ is a more reliable realtime metric of the coupling of feedback from the external cavity to the active region than the LD-internal photodiode signal, $P_{P D}$. Often stray light impinges on the back-facet $P D$ while avoiding the active region (adequate isolation is quite difficult). We show in Fig. 4 that $V_{\mathrm{dc}}$ unlike $P_{P D}$ responds only to dynamics in the active medium. The solid curves in Fig. 4 show (a) $V_{\mathrm{dc}}$ and (b) $P_{\mathrm{PD}}$. In the solid curves, the external cavity is optimally aligned for maximum overlap of the fed back light with the LD active medium. The dashed curves show corresponding data under intentional significant misalignment. Note that in Fig. 4(a) (dashed curve) $V_{\text {dc }}$ remains constant because the misaligned feedback does not affect the LD; whereas Fig. 4(b) (dashed curve) shows that $P_{\mathrm{PD}}$ increases $\sim 1.54 \%$ as the apparent $\eta$ increase when the external cavity is intentionally misaligned. Therefore, $V_{\mathrm{dc}}$ is a more reliable practical probe into the internal dynamics of the ECSL.

Indeed, the current work can be viewed in the broader context where even under sufficient feedback to produce strong chaos, the LD acts as a photodetector as well [17, 18]. But we emphasize that the significance of our work lies in the access to the dynamical-state variable, $N$. Clearly, the approach applies over diverse operating parameter regimes, the subject of future studies.

The authors acknowledge support of the Conseil Regional of Lorraine, GT-CNRS-2958, and National Science Foundation ECCS Grant 0925713.

\section{References}

[1] M. C. Soriano, J. Garca-Ojalvo, C. R. Mirasso, I. Fischer, Rev. Mod. Phys. 85, 421, (2013).

[2] R. F. Kazarinov, R. A. Suris, Zh. Eksp. Teor. Fiz. 66, 1067 (1974) (Sov. Phys.-JETP, 39, 522, (1974)).

[3] Y. Mitsuhashi, J. Shimada, S. Mitsutsuka, IEEE J. Quantum Electron. QE-17, 1216 (1981).

[4] C. Masoller, N. B. Abraham, Phys. Rev. A 57, 1313 (1998).

[5] J. Mork, B. Tromborg, Phys. Rev. Lett. 65, 1999 (1990).

[6] G.H.M. van Tartwijk, G.P. Agrawal, Prog. Quantum Electron. 22, 43 (1998).

[7] D. Brunner, X. Porte, M. C. Soriano, I. Fischer, Sci. Rep. 2, 732 (2012).

[8] J. Ohtsubo, Semiconductor Lasers-Stability, Instability and Chaos, (Springer, Berlin, 2006).

[9] R. Lang, K. Kobayashi, IEEE J. Quantum Electron. 16, 347 (1980).

[10] Y. Mitsuhashi, T. Morikawa, K. Sakurai, A. Seko, J. Shimada, Opt. Commun. 17, 95 (1976).

[11] A. Seko, Y. Mitsuhashi, T. Morikawa, J. Shimada, K. Sakurai, Appl. Phys. Lett. 27, 140 (1975).

[12] W. Ray, W. Lam, P. N. Guzdar, R. Roy, Phys. Rev. E 73, 026219 (2006).

[13] N. M. Al-Hosiny, I. D. Henning, M. J. Adams, IEEE J. Quantum Electron. 42, 570 (2006). 
[14] R. Hui, A. Mecozzi, A. D'Ottavi, P. Spano, Electron. Lett. 26, 997 (1990).

[15] B. Kim, N. Li, A. Locquet, D. S. Citrin, Opt. Expr. 22, 2348 (2014).

[16] See for example J. Piprek, Semiconductor Optoelectronic Devices, (Academic Press, 2003).
[17] Y. Lim, K. Bertling, P. Rio, J. R. Tucker, A. D. Rakic, Photonics: Design, Technology, and Packaging II, 60381-O, (2006)

[18] G. Giuliani, M. Norgia, S. Donati, T. Bosch, J. Opt. A 4, S283 (2002).
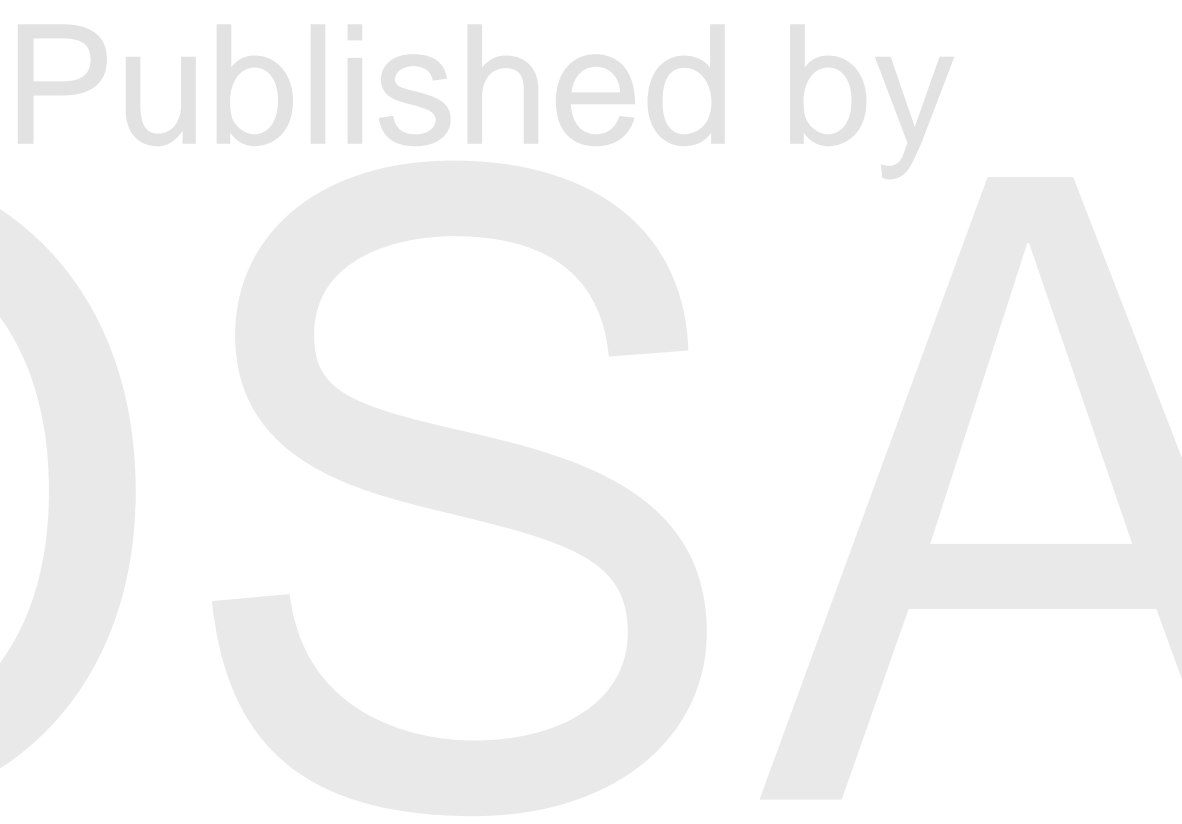